05.4

\title{
Влияние серебра на структуру и эффекты памяти формы биосовместимых сплавов на основе $\mathrm{TiNi}$
}

\author{
(C) Е.С. Марченко, Г.А. Байгонакова , В.Э. Гюнтер \\ Томский государственный университет, Томск, Россия
}

『E-mail: gat27@mail.ru

Поступило в Редакцию 27 марта 2018 г.

Проведено исследование влияния разных концентраций серебра на структуру и эффекты памяти формы биосовместимых сплавов TiNiMoFe. Установлено, что при комнатной температуре все сплавы находятся в смешанном состоянии $\left(B 2+R\right.$ или $\left.B 2+R+B 19^{\prime}\right)$, при этом основной фазой является аустенитная $B 2$-фаза. Показано, что малые концентрации $\mathrm{Ag}$ (до 0.5 at.\%) подавляют эффекты памяти формы, более высокие (1 at.\%) провоцируют зарождение и рост мартенситных кристаллов, при этом увеличивается содержание основной аустенитной $B 2$-фазы и частиц $\mathrm{Ag}$.

DOI: 10.21883/PJTF.2018.16.46482.17309

Биосовместимые многокомпонентные сплавы TiNiMoFe благодаря своим уникальным гистерезисным свойствам вызывают большой научный и практический интерес [1]. Многие характеристики сплавов можно усовершенствовать путем легирования. Перспективным легирующим элементом с биомедицинской точки зрения является серебро, так как оно хорошо известно своим антибактериальным воздействием на клетки [2]. Существует ряд работ [2-6], в которых проведены исследования влияния добавок серебра на свойства сплавов TiNi. B [2-4] рассматриваются антибактериальные свойства тройных сплавов $\mathrm{TiNiAg}$, обусловленные выходом ионов $\mathrm{Ag}^{+}$из фаз чистого серебра на поверхность TiNi. При этом сплавы TiNiAg $\left(C_{\mathrm{Ag}}=1.4-5\right.$ at.\%) показывают хорошую биосовместимость и коррозионную стойкость, сравнимую с таковой для бинарных сплавов TiNi. Кроме антибактериальных свойств в $[5,6]$ исследовано влияние добавок серебра $\left(C_{\mathrm{Ag}}=0.6-1.3,1.7\right.$ и 1.9 at.\%) на гистерезисные и физико-механические характеристики. 
С целью расширения полезных свойств биомедицинских сплавов TiNiMoFe c эффектом памяти формы (ЭПФ) проведено их объемное легирование серебром. В работе приведены экспериментальные исследования влияния разных концентраций серебра на структуру и параметры многократного ЭПФ сплавов TiNiMoFe.

Сплавы TiNiMoFeAg были выплавлены в индукционной печи ИСВ-0.004 ПИ М1 в атмосфере инертного газа аргона по следующей схеме легирования: $\mathrm{Ti}_{50} \mathrm{Ni}_{49.5-x} \mathrm{Mo}_{0.3} \mathrm{Fe}_{0.2} \mathrm{Ag}_{x}$ ( $x=0,0.1,0.5,1$ at.\%). Из полученных слитков без дополнительных термомеханических обработок на электроэрозионной установке были вырезаны образцы длиной $50 \mathrm{~mm}$ с площадью поперечного сечения $1 \mathrm{~mm}^{2}$. Параметры ЭПФ исследовались в условиях растяжения под постоянной нагрузкой $2 \mathrm{~kg}$. Изучение фазового состава и структурных параметров образцов проводилось на дифрактометре XRD-6000 в $\mathrm{Cu} K_{\alpha}$-излучении. Методом дифракции отраженных электронов на системе с электронным и сфокусированными пучками QUANTA 200 3D был выполнен энергодисперсионный анализ матриц сплавов.

Методом рентгеноструктурного анализа проведено исследование пяти поликристаллических образцов сплавов TiNiMoFeAg с разным содержанием Ag. Качественный и количественный фазовый состав, параметры решеток, кристаллические области когерентного рассеяния $(\mathrm{OKР})$ и микродеформация решеток $(\Delta d / d)$ фаз приведены в таблице. Установлено, что при комнатной температуре все сплавы находятся в смешанном состоянии $\left(B 2+R\right.$ или $\left.B 2+R+B 19^{\prime}\right)$, при этом основной фазой является аустенитная $B 2$-фаза с ОЦК-решеткой. С увеличением содержания $\mathrm{Ag}$ массовая доля $B 2$-фазы растет, что сопровождается изменением параметра ОЦК-решетки в результате введения атомов Ag в элементарную ячейку B2-фазы. Уширение и закономерное расщепление дифракционных отражений $B 2$-фазы: [110], [200], [211] — свидетельствуют о наличии ромбоэдрической $R$-фазы. Это хорошо согласуется с данными исследований удельного электросопротивления $\rho(T)$ на этой же серии сплавов [7]. Также на рентгенограммах всех сплавов обнаружены дифракционные линии фазы $\mathrm{Ti}_{2} \mathrm{Ni}$ с высокой интенсивностью, рост концентрации серебра приводит к снижению интенсивностей дифракционных пиков от фазы $\mathrm{Ti}_{2} \mathrm{Ni}$. В сплавах с 0 и $0.1 \mathrm{at} \% \mathrm{Ag}$ присутствуют слабые структурные линии моноклинной мартенситной фазы $B 19^{\prime}$ с массовой долей не более $5 \%$. Серебро кристаллизуется в матрице TiNi в чистом виде и имеет ГЦК-структуру, малая часть $\mathrm{Ag}$

7 Письма в ЖТФ, 2018, том 44, вып. 16 


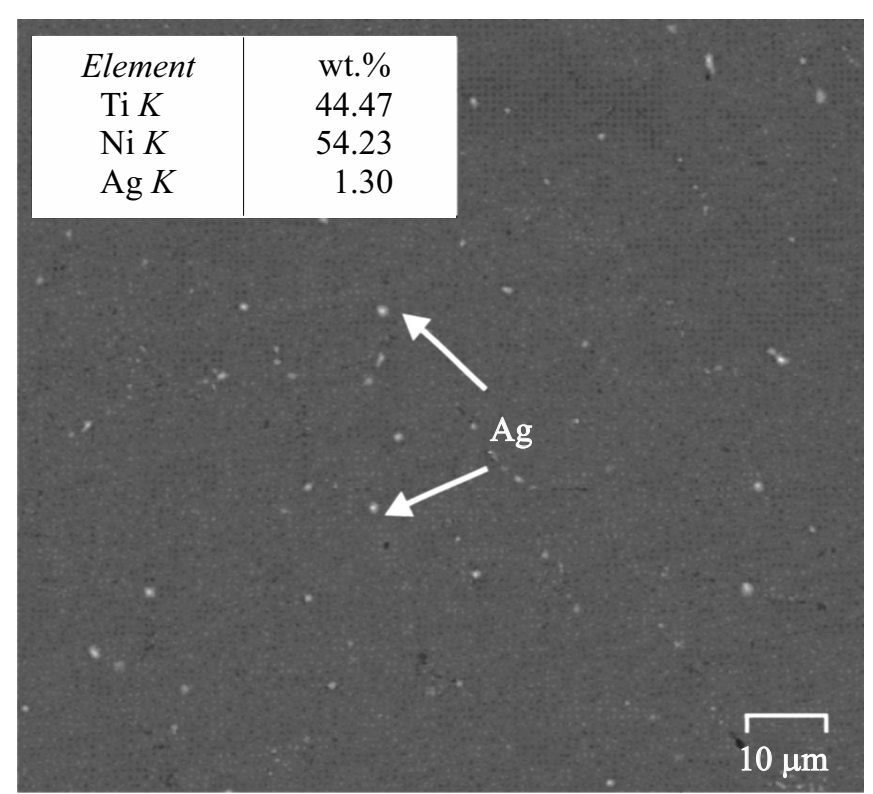

Рис. 1. Результаты энергодисперсионного анализа матрицы сплавов TiNiMoFeAg c 1 at.\% Ag.

(до 0.2 at.\%) растворяется в фазе B2, т.е. замещает атомы основных элементов в кристаллической решетке. Это подтверждают данные локального энергодисперсионного микроанализа на рис. 1 (вставка), где приведены результаты количественного элементного микроанализа матрицы сплава.

Небольшие концентрации серебра увеличивают преимущественную ориентацию кристаллической решетки зерен $B 2$-фазы в направлении [200] с 37 до 74\% (при 0.2 at.\%). Это указывает на то, что даже незначительное изменение состава приводит к росту внутренних напряжений в зернах B2-фазы, что хорошо согласуется с ростом значений упругих внутренних напряжений второго рода $\Delta d / d$ внутри кристаллической ячейки $B 2$-фазы (с $0.54 \cdot 10^{-3}$ до $3.27 \cdot 10^{-3}$ ). Кроме того, частицы чистого серебра также могут способствовать появлению дополнительных источников внутренних напряжений в материале, ко-

Письма в ЖТФ, 2018, том 44, вып. 16 
Данные рентгенографического исследования сплавов TiNiMoFeAg

\begin{tabular}{|c|c|c|c|c|c|}
\hline Образец & $\begin{array}{c}\text { Обнаруженные } \\
\text { фазы }\end{array}$ & $\begin{array}{l}\text { Содержание } \\
\text { фаз, mass\% }\end{array}$ & $\begin{array}{l}\text { Параметры } \\
\text { решетки, } \AA\end{array}$ & $\begin{array}{l}\text { Размер } \\
\text { OKР, nm }\end{array}$ & $\Delta d / d, 10^{-3}$ \\
\hline 0 at. $\% \mathrm{Ag}$ & $\begin{array}{c}\operatorname{TiNi}(B 2) \\
37 \% \text { вдоль }[200] \\
\mathrm{Ti}_{2} \mathrm{Ni} \text { (trigonal) } \\
\mathrm{TiNi}\left(B 19^{\prime}\right) \\
\\
R\end{array}$ & Следы & $\begin{array}{l}a=3.0111 \\
a=11.2755 \\
a=4.6190 \\
b=4.1201 \\
c=2.903 \\
a=9.0316\end{array}$ & $\begin{array}{l}19 \\
25 \\
-\end{array}$ & $\begin{array}{c}0.54 \\
2.24 \\
-\end{array}$ \\
\hline 0.1 at. $\% \mathrm{Ag}$ & $\begin{array}{c}\operatorname{TiNi}(\text { В2) } \\
48 \% \text { вдоль }[200] \\
\mathrm{Ti}_{2} \mathrm{Ni} \text { (trigonal) } \\
\mathrm{TiNi}\left(\text { В } 19^{\prime}\right) \\
\\
R \\
\mathrm{Ag} \text { (cubic) }\end{array}$ & $\begin{array}{l}\text { Следы } \\
\text { Следы }\end{array}$ & $\begin{array}{l}a=3.0171 \\
a=11.2209 \\
a=4.6175 \\
b=4.125 \\
c=2.902 \\
a=9.0419 \\
a=4.0756\end{array}$ & $\begin{array}{l}28 \\
15 \\
-\end{array}$ & $\begin{array}{c}1.44 \\
2.35 \\
-\end{array}$ \\
\hline 0.5 at. $\% \mathrm{Ag}$ & $\begin{array}{c}\mathrm{TiNi}(B 2) \\
\mathrm{Ti}_{2} \mathrm{Ni} \text { (trigonal) } \\
\mathrm{Ag} \text { (cubic) } \\
R\end{array}$ & $\begin{array}{c}87 \\
13 \\
\text { Следы } \\
\text { Следы }\end{array}$ & $\begin{array}{l}a=2.9946 \\
a=11.2503 \\
a=4.0227 \\
a=9.05326\end{array}$ & $\begin{array}{l}17 \\
11 \\
- \\
-\end{array}$ & $\begin{array}{c}2.01 \\
2.58 \\
- \\
-\end{array}$ \\
\hline 1 at. $\% \mathrm{Ag}$ & $\begin{array}{c}\mathrm{TiNi}(B 2) \\
\mathrm{Ti}_{2} \mathrm{Ni} \text { (trigonal) } \\
\mathrm{Ag} \text { (cubic) } \\
R\end{array}$ & $\begin{array}{c}89 \\
6 \\
5 \\
\text { Следы }\end{array}$ & $\begin{array}{l}a=3.0196 \\
a=11.2588 \\
a=4.0845 \\
a=9.08633\end{array}$ & $\begin{array}{l}31 \\
13 \\
- \\
-\end{array}$ & $\begin{array}{c}3.27 \\
2.43 \\
- \\
-\end{array}$ \\
\hline
\end{tabular}

торые, с одной стороны, могут стимулировать начало мартенситного превращения (МП), а с другой - быть барьерами, препятствующими движению межфазной границы в процессе фазового перехода.

Рассмотрим влияние серебра на параметры многократного ЭПФ сплавов TiNiMoFeAg (рис. 2). Реализация ЭПФ в сплавах TiNiMoFeAg сильно зависит от количества легирующей добавки, и при различных концентрациях серебра в процесс фазового перехода включаются разные факторы. Изменение объема, размеров зерен матричной фазы и кристаллизация вторичных фаз с последующим изменением соотно-

7* Письма в ЖТФ, 2018, том 44, вып. 16 

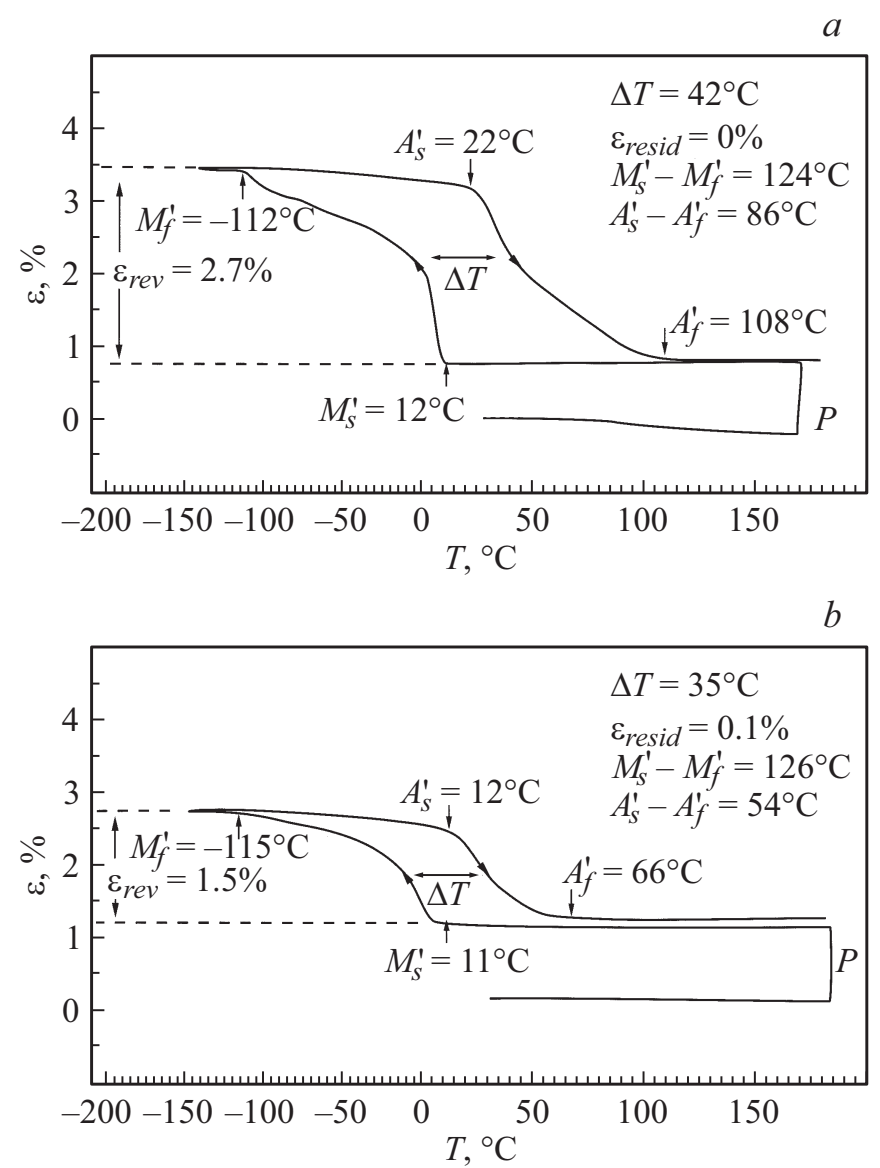

Рис. 2. Влияние легирования серебром на многократный эффект памяти формы в сплавах TiNiMoFeAg с $C_{\mathrm{Ag}}=0(a), 0.1(b), 0.5(c)$ и 1 at.\% (d) при постоянной нагрузке $P=2 \mathrm{~kg}$. $M_{s}^{\prime}, M_{f}^{\prime}$ - температуры начала и конца прямого МП, $A_{s}^{\prime}, A_{f}^{\prime}$ - температуры начала и конца обратного МП, $M_{s}^{\prime}-M_{f}^{\prime}-$ температурный интервал прямого МП, $A_{s}^{\prime}-A_{f}^{\prime}$ - температурный интервал обратного МП.

шения Тi и $\mathrm{Ni}$ в матрице сильно влияют на смещение температур МП. Малые концентрации Ag (до 0.5 at.\%) приводят к снижению 

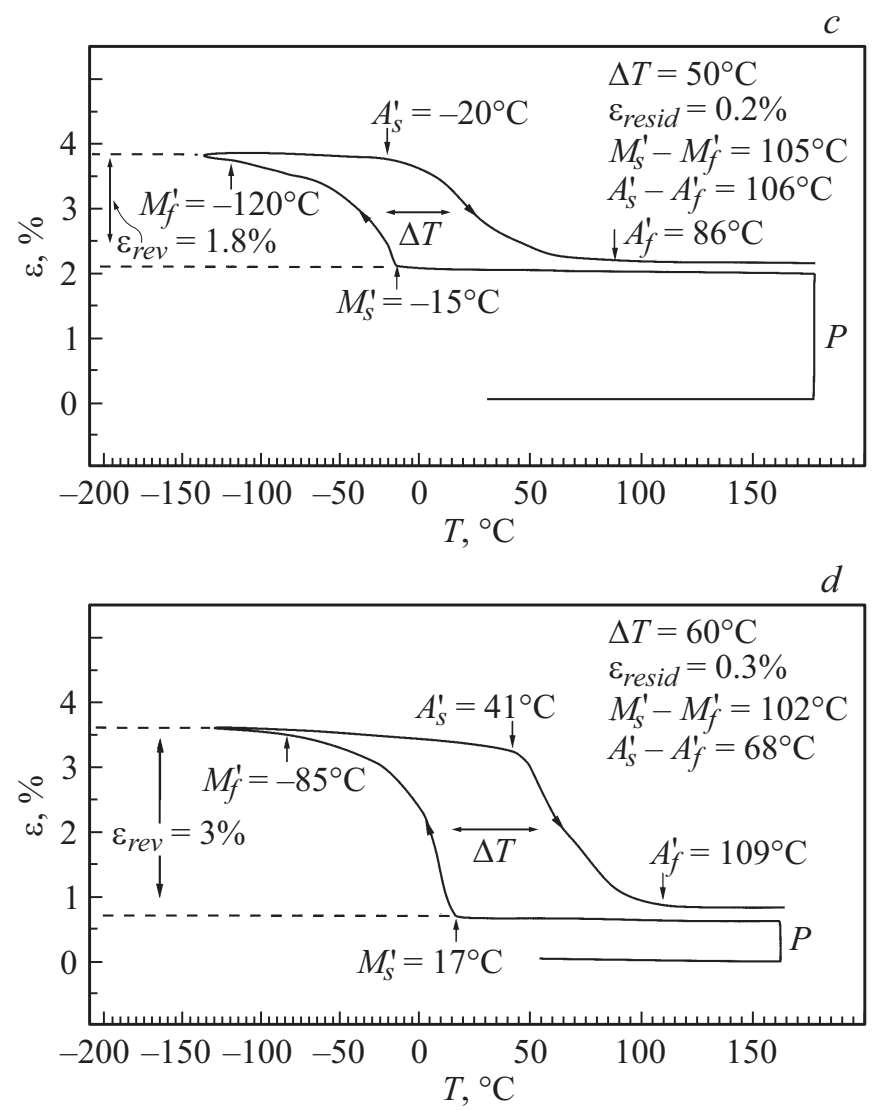

Рис. 2 (продолжение).

характеристических температур начала прямого МП $M_{s}$ под нагрузкой из-за появления и накопления частиц фазы $\mathrm{Ag}$, которые препятствуют движению межфазной границы и стабилизируют B2-фазу. С повышением концентрации $\mathrm{Ag}$ до $1 \mathrm{at} \%$ доминирующим фактором становится рост полей внутренних напряжений, образованных частицами $\mathrm{Ag}$, и в соответствии с уравнением Клапейрона-Клаузиуса [8] происходит повышение температуры $M_{s}$, облегчается зарождение кристаллов мартенситной фазы.

Письма в ЖТФ, 2018, том 44, вып. 16 
Различные структурные состояния, связанные с введением легирующего элемента, изменяют такие параметры ЭПФ, как ширина петли гистерезиса $\Delta T$, общая накопленная деформация $\varepsilon_{r e v}$, остаточная деформация $\varepsilon_{\text {resid }}$.

B сплавах на основе TiNi MП в термодинамическом смысле всегда сопровождается диссипацией энергии и производством энтропии [9]. Ширина петли гистерезиса $\Delta T$ соответствует количеству рассеянной энергии при полном цикле МП $(A \rightarrow M$ и $M \rightarrow A)$. Из зависимостей накопления и возврата деформации при многократном ЭПФ (рис. 2) видно, что увеличение концентрации легирующего элемента до 1 at.\% вызывает уширение $\Delta T$ (от 40 до $60^{\circ} \mathrm{C}$ ), так как из-за структурного усложнения материала вторичными фазами в процессе фазового перехода $B 2 \leftrightarrow R \leftrightarrow B 19^{\prime}$ происходит большее рассеяние энергии на дефектах или частицах фазы $\mathrm{Ag}$, массовая доля которых тоже увеличивается (до 5 mass \%).

Частицы серебра оказывают влияние и на накопление деформации. Деформация сплавов с ЭПФ представляет собой комплексную величину, состоящую из упругой, мартенситной и пластической деформации. Легирование малыми концентрациями $\mathrm{Ag}$ (до 0.5 at.\%) резко снижает величину $\varepsilon_{\text {rev }}$. Появление в сплаве частиц $\mathrm{Ag}$ провоцирует скопление локальных напряжений возле частиц $\mathrm{Ag}$, что приводит к большему накоплению пластической составляющей деформации и, как следствие, к уменьшению величины накопленной мартенситной и общей деформации $\varepsilon_{r e v}$. Большие добавки Ag (от 1 at.\%) увеличивают $\varepsilon_{r e v}$ до величины, большей, чем у исходного сплава. Это связано с ростом массовой доли B2-фазы, ответственной за накопление мартенситной деформации до $\sim 90 \%$ (см. таблицу).

Увеличение содержания $\mathrm{Ag}$ в сплаве сопровождается ростом величины остаточной деформации $\varepsilon_{\text {resid }}$, которая характеризует долю пластической деформации. Образованные в процессе легирования некогерентные фазы $\mathrm{Ag}$ являются препятствиями для реализации МП, преодоление которых при деформации происходит за счет пластического сдвига, и соответственно увеличивается доля пластической составляющей (остаточной деформации $\varepsilon_{\text {resid }}$ ) в величине общей накопленной деформации $\varepsilon_{\text {rev }}$.

Таким образом, параметры ЭПФ в сплавах TiNiMoFeAg являются чувствительными к малейшим изменениям структурно-фазового состава сплава. Серебро структурно усложняет матрицу сплава частицами $\mathrm{Ag}$

Письма в ЖТФ, 2018, том 44, вып. 16 
и включается в процесс фазового перехода $B 2 \leftrightarrow R \leftrightarrow B 19^{\prime}$. Малые концентрации $\mathrm{Ag}$ (до 0.5 at.\%) подавляют ЭПФ, более высокие (1 at.\%) провоцируют зарождение и рост мартенситных кристаллов, при этом увеличивается содержание основной аустенитной $B 2$-фазы и частиц $\mathrm{Ag}$. Отдельные частицы фаз $\mathrm{Ag}$ в матрице сплавов становятся дополнительными источниками полей внутренних напряжений, что приводит к росту пластической составляющей деформации. Также влияние серебра проявляется в росте напряжений и изменении сил межатомного взаимодействия в структуре $B 2$.

Работа выполнена в рамках программы повышения конкурентоспособности ТГУ Фонда им. Д.И. Менделеева (2018 г.).

\section{Список литературы}

[1] Ходоренко В.Н., Кафтаранова М.И., Гюнтер В.Э. // Письма в ЖТФ. 2015. T. 41. B. 6. C. $81-89$.

[2] Melaiye A., Youngs W.J. // Expert Opin. Ther. Pat. 2005. V. 15. P. 125-130.

[3] Zheng Y.F., Zhang B.L., Wang B.L., Wang Y.B., Li L., Yang Q.B., Cui L.S. // Acta Biomater. 2011. V. 7. N 6. P. 2758-2767.

[4] Jhou W.-T., Wang C., Ii S., Chiang H.-S., Hsueh C.-H. // J. Alloys Compd. 2018. V. 738. P. 336-344.

[5] Chun S.-J., Noh J.-P., Yeom J.-T., Kim J.-I., Nam T.-H. // Intermetallics. 2014. V. 46. P. 91-96.

[6] Alvares da Silva G., Otubo J. // MATEC Web Conf. 2015. V. 33. P. 03009.

[7] Gunther V., Marchenko E., Chekalkin T., Baigonakova G., Kim J.-S., Kang J.-H., Klopotov A. // Mater. Res. Express. 2017. V. 4. N 10. P. 105702.

[8] Liu Y., Yang H. // Smart Mater. Struct. 2007. V. 16. N 1. P. 22-27.

[9] Otsuka K., Saxena A., Deng J., Ren X. // Phil. Mag. 2011. V. 91. N 36. P. 4514-4535.

Письма в ЖТФ, 2018, том 44, вып. 16 